University of Nebraska - Lincoln

DigitalCommons@University of Nebraska - Lincoln

Faculty Publications - Chemistry Department Published Research - Department of Chemistry

8-21-2019

\title{
A Panel of Protein Kinase Chemosensors Distinguishes Different Types of Fatty Liver Disease
}

Jon R. Beck

Fatima Cabral

Karuna Rasineni

Carol A. Casey

Edward N. Harris

See next page for additional authors

Follow this and additional works at: https://digitalcommons.unl.edu/chemfacpub

Part of the Analytical Chemistry Commons, Medicinal-Pharmaceutical Chemistry Commons, and the Other Chemistry Commons

This Article is brought to you for free and open access by the Published Research - Department of Chemistry at DigitalCommons@University of Nebraska - Lincoln. It has been accepted for inclusion in Faculty Publications -Chemistry Department by an authorized administrator of DigitalCommons@University of Nebraska - Lincoln. 


\section{Authors}

Jon R. Beck, Fatima Cabral, Karuna Rasineni, Carol A. Casey, Edward N. Harris, and Cliff I. Stains 


\title{
A Panel of Protein Kinase Chemosensors Distinguishes Different Types of Fatty Liver Disease
}

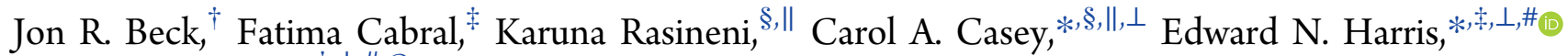
and Cliff I. Stains ${ }^{*},+,, \#, @ \circledast$

${ }^{\dagger}$ Department of Chemistry, University of Nebraska-Lincoln, Lincoln, Nebraska 68588, United States

${ }^{\ddagger}$ Department of Biochemistry, University of Nebraska-Lincoln, Lincoln, Nebraska 68588, United States

${ }^{\S}$ Division of Gastroenterology-Hepatology, University of Nebraska Medical Center, Omaha, Nebraska 68198, United States

"Research Service, Veterans' Affairs, Nebraska-Western Iowa Health Care System, Omaha, Nebraska 68105, United States

${ }^{\perp}$ Nebraska Center for Integrated Biomolecular Communication, University of Nebraska-Lincoln, Lincoln, Nebraska 68588, United States

\#Cancer Genes and Molecular Regulation Program, Fred \& Pamela Buffet Cancer Center, University of Nebraska Medical Center, Omaha, Nebraska 68198, United States

${ }^{\circledR}$ Department of Chemistry, University of Virginia, Charlottesville, Virginia 22904, United States

Supporting Information

ABSTRACT: The worldwide incidence of fatty liver disease continues to rise, which may account for concurrent increases in the frequencies of more aggressive liver ailments. Given the existence of histologically identical fatty liver disease subtypes, there is a critical need for the identification of methods that can classify disease and potentially predict progression. Herein, we show that a panel of protein kinase chemosensors can distinguish fatty liver disease subtypes. These direct activity measurements highlight distinct differences between histologically identical fatty liver diseases arising from diets rich in fat versus alcohol and identify a previously unreported decrease in p38 $\alpha$ activity associated with a high-fat diet. In addition, we have profiled kinase activities in both benign (dietinduced) and progressive (STAM) disease models. These experiments provide temporal insights into kinase activity during disease development and progression. Altogether, this work provides the basis for the future development of clinical diagnostics and potential treatment strategies.

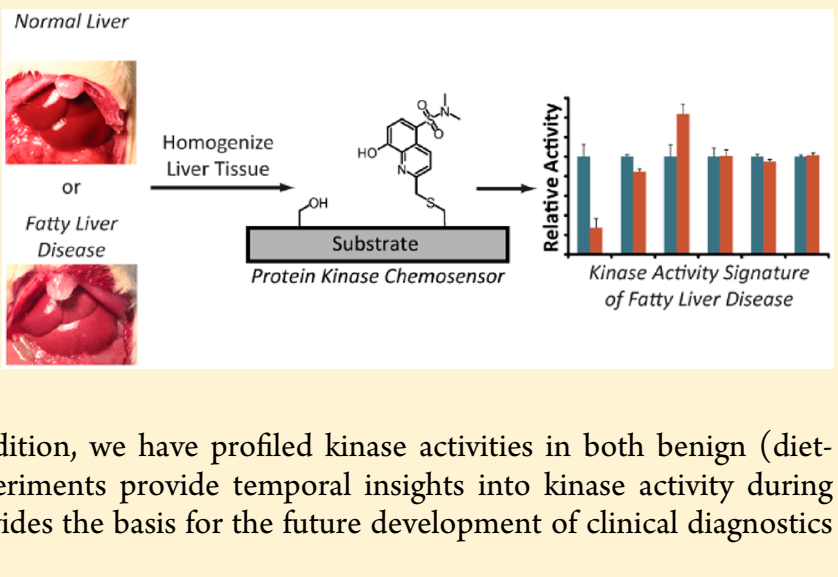

$\mathrm{N}$ onalcoholic fatty liver disease (NAFLD) is a metabolic spectrum disorder characterized by accumulation of $>5 \%$ fat by weight in the liver. ${ }^{1}$ This disease spectrum is characterized by morphological changes in the liver such as accumulation of fat associated with inflammation, known as nonalcoholic steatohepatitis (NASH), and the eventual development of fibrosis followed by hepatocellular carcinoma (HCC) (Figure 1A). ${ }^{2}$ Worldwide NAFLD incidence has reached alarming levels, with an estimated $25 \%$ of the population afflicted by this disease (24\% in the United States), with the highest incidences in the Middle East (32\%) and lowest incidences in Africa (14\%). ${ }^{3}$ The projected direct medical cost in the United States alone on a per annual basis is predicted to be $\$ 103$ billion. ${ }^{4}$ Diagnosis of NAFLD can be complicated due to the existence of a histologically identical disease known as alcoholic fatty liver disease (AFLD)., Though histologically identical, these two diseases arise from significantly different behaviors, namely, the consumption of alcohol (AFLD) or high-calorie diets rich in sugar, oils, and fats (NAFLD). Unfortunately, the clinical definition of significant alcohol consumption remains controversial; ${ }^{6}$ leading to issues in distinguishing AFLD versus NAFLD based solely on histology and self-reported alcohol consumption. Thus, methodologies capable of parsing NAFLD from AFLD are needed. In this regard, one-step direct activity assays provide increased throughput and reduced assay time compared to traditional approaches such as Western blotting. Ultimately, such relatively low-cost and rapid analysis techniques could also be utilized in the clinical setting to inform personalized treatment strategies.

While simple steatosis in NAFLD is generally benign, a growing body of evidence suggests that individuals with NASH are at higher risk for the development of nonviral HCC. Indeed, recent statistics indicate dramatic increases in both liver cancer rates and deaths in the United States. ${ }^{8,9}$ One

Received: June 26, 2019

Revised: August 13, 2019

Published: August 21, 2019 
A

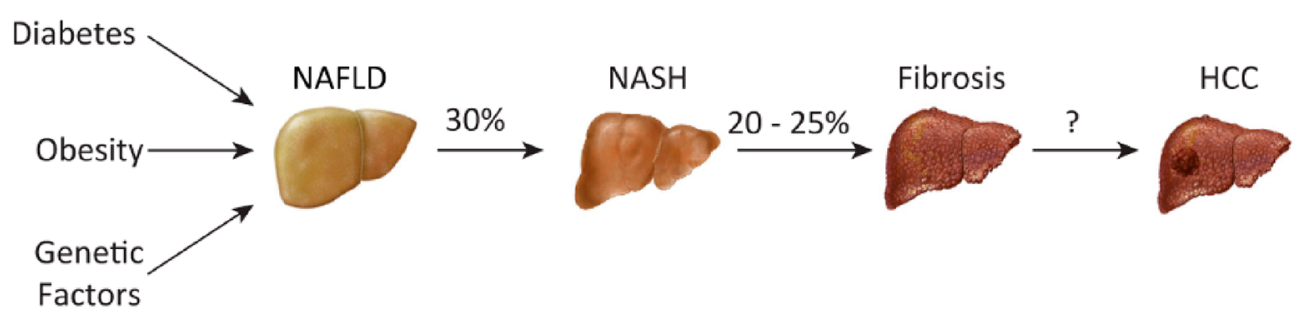

B

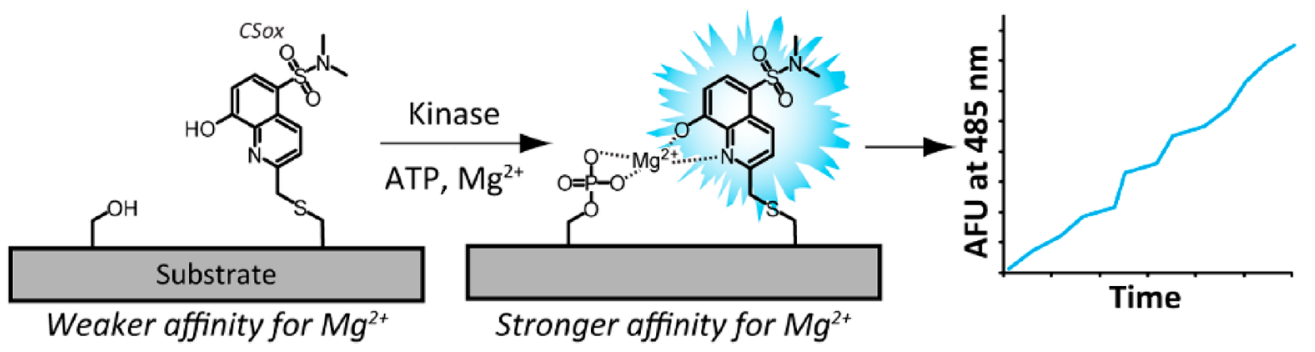

C

\begin{tabular}{ccc} 
Kinase & Substrate Sequence & Pathway \\
\hline p38 $\alpha$ & RKPDLRVVIPP-(AOO) ${ }_{3}$-QP-CSox-ASPVV & Inflammation \\
MK2 & AHLQRQLSI-CSox-HH & Inflammation \\
JNK1/2/3 & ERPSRDHLYLPLEP-(AOO) ${ }_{2}$-SANLLSP-CSox-PA & Inflammation \\
ERK1/2 & VP-CSox-LTPGGRRG-PNT Domain (S46-K138) & Growth \\
Akt & ARKRERAYSF-CSox-HHA & Survival \\
PKA & ALRRASL-CSox-AA & Metabolism \\
\hline
\end{tabular}

D

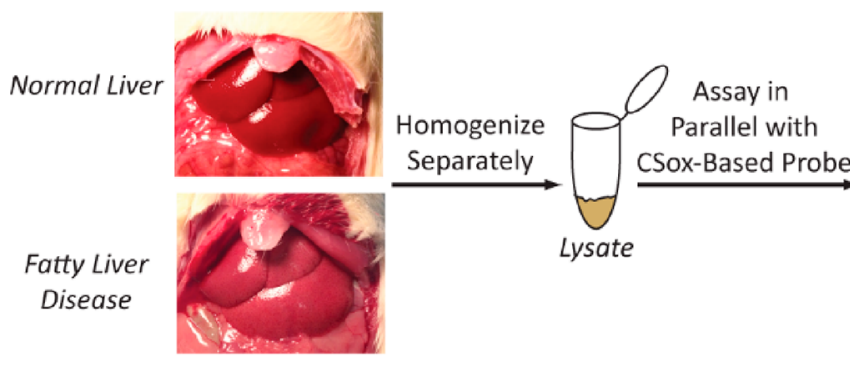

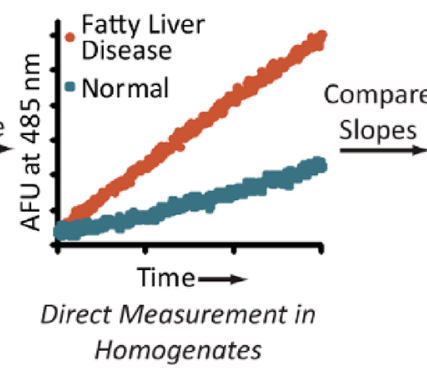

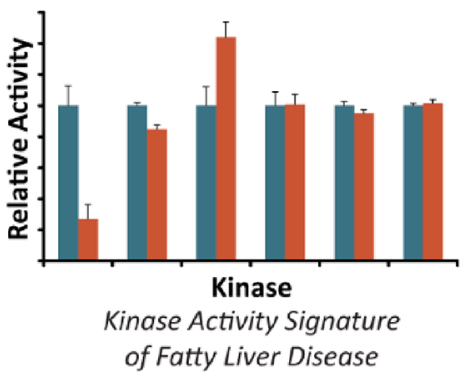

Figure 1. Profiling changes in fatty liver signaling using a panel of validated CSox activity probes for protein kinases. (A) Although several factors contribute to the development of NAFLD, the common histological outcome is accumulation of fat in the liver. In certain patients with underlying metabolic disorders, NAFLD can progress to more aggressive disease states such as NASH, fibrosis, and ultimately HCC. The estimated percentage of patient progression is given above the arrows, adapted with permission from ref 2. (B) A nonphosphorylated CSox substrate has a low affinity for $\mathrm{Mg}^{2+}$ and weak fluorescence (left). Upon the addition of a phosphate by a kinase, the affinity of the sensor for $\mathrm{Mg}^{2+}$ increases, leading to a concurrent increase in fluorescence (middle). This increase in fluorescence can be plotted as a function of time and is proportional to the enzymatic activity of the kinase (right). (C) Panel of CSox-based activity sensors that have been validated for the indicated kinase in unfractionated lysates obtained from liver hepatocytes. In certain cases, probes contain synthetic linkers (AOO, 8-amino-3,6-dioxaoctanoic acid) or protein domains (PNT domain S46-K138). Canonical pathways are given, and CSox is colored blue with the site of phosphorylation chlored red. (D) Workflow for the analysis of kinase activities in normal vs fatty livers. Liver samples are homogenized and normalized to protein content. Homogenates are then run in parallel against the panel of kinase activity probes, and the activities of each kinase can then be compared in normal (blue) and fatty liver (red) samples to provide a signature of the disease state.

potential cause of this observation is the progression of NAFLD to HCC (Figure 1A). Due to the prevalence of NAFLD in the general population, there is also a need for the identification of markers associated with disease progression. $^{10,11}$

To begin to address these issues, we hypothesized that alterations in protein kinase activity could be used to distinguish NAFLD from AFLD and provide insights into pathways associated with disease progression. Several elegant chemical biology tools have been developed to probe kinase function and activity in biological samples. ${ }^{12-19}$ Here, we chose to employ a panel of direct, kinase chemosensors based on the phosphorylation-sensitive sulfonamido-oxine (Sox) fluorophore developed by the Imperiali laboratory. ${ }^{20,21}$ 
Importantly, Sox-based probes are capable of directly reporting on kinase enzymatic activity in real-time using a straightforward fluorescence readout in unfractionated tissue homogenates. $^{22,23}$ Sox displays chelation-enhanced fluorescence (CHEF) upon binding to $\mathrm{Mg}^{2+}$. Proximal enzymatic installation of a phosphoryl group in a peptide sequence labeled with Sox dramatically increases the affinity of the Soxcontaining peptide for $\mathrm{Mg}^{2+}$. Thus, Sox peptides can be used to directly visualize protein kinase enzymatic activity through CHEF (Figure 1B). First-generation Sox sensors relied upon a $\beta$-turn motif to correctly orient the Sox fluorophore and the site of phosphorylation for subsequent $\mathrm{Mg}^{2+}$ binding. More recently, second-generation Sox sensors that incorporate a single unnatural amino acid mutation consisting of a cysteine residue alkylated with the Sox fluorophore have been developed (CSox) (Figure 1B). ${ }^{24}$ When placed proximal to a site of phosphorylation ( \pm 2 residues), CSox allows for CHEF upon phosphorylation, enabling the design of more efficient kinase substrates through incorporation of both $\mathrm{N}$ - and $\mathrm{C}$ terminal recognition elements. Panels of CSox-based probes have been utilized to fingerprint mixtures of mitogen-activated protein kinases (MAPKs) in vitro. ${ }^{25}$ Additional selectivity for a kinase can be imparted through the incorporation of docking domains to drive binding affinity and/or the use of inhibitors to suppress off-target activity. ${ }^{22,23}$ Indeed, these approaches have yielded validated probes for $\mathrm{p} 38 \alpha, \mathrm{MK} 2, \mathrm{JNK} 1 / 2 / 3$, ERK1/2, Akt, and PKA that can be used to selectively report on kinase activity in unfractionated lysates obtained from liver hepatocytes (Figure 1C). ${ }^{26-28}$ Inspired by previous work demonstrating the ability to fingerprint biological samples based on kinase activity, ${ }^{29}$ we show that NAFLD and ALFD can be parsed on the basis of differences in kinase activity as assessed by this panel of CSox-based chemosensors. In addition, we identify factors that may distinguish benign and progressive NAFLD as well as provide a temporal map of kinase activity during progression of NAFLD to HCC (Figure 1D). These results provide a rapid means to distinguish between histologically identical fatty liver disease subtypes and could be used as the basis for the development of diagnostic tools.

\section{MATERIALS AND METHODS}

General Reagents and Procedures. All assays were performed in 384-well, white, half-area plates (Corning, 3824) with a total volume of $40 \mu \mathrm{L}$. Fluorescence data were collected with a Bio-Tek Synergy H1 microplate reader with excitation at $360 \mathrm{~nm}$ and emission at $485 \mathrm{~nm}$. Assay buffer consisted of $50 \mathrm{mM}$ Tris- $\mathrm{HCl}\left(\mathrm{pH} 7.5\right.$ at $\left.22{ }^{\circ} \mathrm{C}\right), 10 \mathrm{mM} \mathrm{MgCl}_{2}, 1 \mathrm{mM}$ EGTA, $2 \mathrm{mM}$ DTT, 0.01\% Brij-35-P, and $1 \mathrm{mM}$ ATP. Lysis buffer consisted of $50 \mathrm{mM}$ Tris- $\mathrm{HCl}\left(\mathrm{pH} 7.5\right.$ at $\left.22{ }^{\circ} \mathrm{C}\right), 150$ $\mathrm{mM} \mathrm{NaCl}, 30 \mathrm{mM} \mathrm{NaF}, 1 \%$ (v/v) Triton X-100, 2 mM EGTA, $1 \mathrm{mM} \mathrm{DTT}, 1 \%(\mathrm{v} / \mathrm{v})$ protease inhibitor cocktail III (EMD Millipore, 539134), and $1 \%(\mathrm{v} / \mathrm{v})$ phosphatase inhibitor cocktail III (Sigma, P0044). Detailed methods for probe synthesis as well as tissue lysis and analysis can be found in previously published protocols, ${ }^{22,23}$ brief descriptions of these procedures are given below.

Probe Synthesis. All peptide sensors were synthesized using standard Fmoc-protected solid-phase peptide synthesis methods as previously reported. ${ }^{23}$ Sox was introduced either through direct alkylation of on-resin peptides or through incorporation of Fmoc-CSox. Peptide sensors were cleaved from the resin and purified by high-performance liquid chromatography, and their identity was verified by mass spectrometry. The ERK1/2 sensor was prepared as described previously, ${ }^{26}$ purified by FPLC, and confirmed by sodium dodecyl sulfate-polyacrylamide gel electrophoresis (SDSPAGE).

Animal Models. For the NAFLD model, simple steatosis was generated by feeding $200 \mathrm{~g}$ male Wistar rats with a high-fat diet consisting of $60 \%$ calories from saturated, monounsaturated, and polyunsaturated fatty acids (ResearchDiets, D08060104) ad libitum for 8 weeks. ${ }^{5}$ To generate their respective controls, rats of the same age and weight were fed a control diet consisting of $10 \%$ calories from fatty acids (Research Diets, D12450K) ad libitum for 8 weeks. For the AFLD model, male Wistar rats were pair-fed with LieberDeCarli control or EtOH-liquid diets that contained calories of $18 \%$ from protein, $35 \%$ from fat, $11 \%$ from carbohydrate, and $36 \%$ from ethanol. ${ }^{5}$ In the control diet, ethanol was replaced isocalorically with maltodextrin.

Frozen STAM mouse tissue samples were obtained from SMC Laboratories (Tokyo, Japan). In-house STAM tissues were generated by injecting 2-day-old pups with a single bolus of $0.2 \mathrm{mg}$ of STZ subcutaneously. Once the mice were weaned, they were immediately fed a high-fat diet ad libitum (BioServ F3282) until age 6 weeks (simple steatotis), 8 weeks (NASH), 12 weeks (fibrosis), or 20 weeks (HCC). Uninjected littermates were fed an isocaloric low-fat control diet (BioServ F4031) over the same time courses. ${ }^{30}$

All animal protocols were reviewed by the Institutional Animal Care and Use Committees (IACUCs) of both the University of Nebraska-Lincoln and Nebraska-Western Iowa Health Care System Research Service, both of which have been certified by the American Association for the Accreditation of Laboratory Animal Care (AAALAC).

Generation of Tissue Homogenates. Tissue homogenates were prepared as described previously. ${ }^{22}$ Frozen liver tissue samples were weighed and rinsed three times with icecold phosphate-buffered saline. Ice-cold lysis buffer $(3 \mu \mathrm{L} / \mathrm{mg}$ of tissue) was added to each sample. Tissue samples were then homogenized using a hand-held homogenizer (Omni, TH115PCRH) with disposable, hard tissue tips (Omni, 30750H). Samples were incubated on ice for $15 \mathrm{~min}$ followed by centrifugation at $4{ }^{\circ} \mathrm{C}$ for $5 \mathrm{~min}$. The soluble fraction of each homogenate was aliquoted, flash-frozen in liquid nitrogen, and stored at $-80{ }^{\circ} \mathrm{C}$. The total protein content in each sample was determined using the Bio-Rad Protein Assay Kit (Bio-Rad, 5000001) according to the manufacturer's protocol.

Kinase Activity Assays. Assays were conducted as described previously. ${ }^{22}$ Assays were corrected for lag times, and slopes were calculated using the linear region of the data. While lag times and linear regions vary between kinases, conditions for individual kinases were identical allowing comparison of relative activities (slopes). Each kinase was assayed in duplicate for each animal in assay buffer using the indicated Sox sensor for Akt $(2.5 \mu \mathrm{M})$, ERK1/2 $(5 \mu \mathrm{M})$, MK2 $(2.5 \mu \mathrm{M}), \mathrm{p} 38 \alpha(1 \mu \mathrm{M})$, or JNK $(3 \mu \mathrm{M})$. The assays were miniaturized for use in 384-well plates using a total volume of $40 \mu \mathrm{L}$ per well. Total protein amounts for each probe were as follows: $3.3 \mu \mathrm{g}$ for Akt, MK2, and p38 $\alpha$; $6.6 \mu \mathrm{g}$ for JNK1/2/3 and PKA; and $13.3 \mu \mathrm{g}$ for ERK1/2. Inhibitors were included in Akt ( $4 \mu \mathrm{M}$ PKC inhibitor peptide, $4 \mu \mathrm{M}$ calmidazolium, and 5 $\mu \mathrm{M}$ GF109203X), p38 $\alpha$ (10 $\mu \mathrm{M}$ staurosporine), and PKA (4 $\mu \mathrm{M}$ PKC inhibitor peptide, $4 \mu \mathrm{M}$ calmidazolium, and $5 \mu \mathrm{M}$ GF109203X) assays. The activity of $\mathrm{p} 38 \alpha$ was resolved by 
A

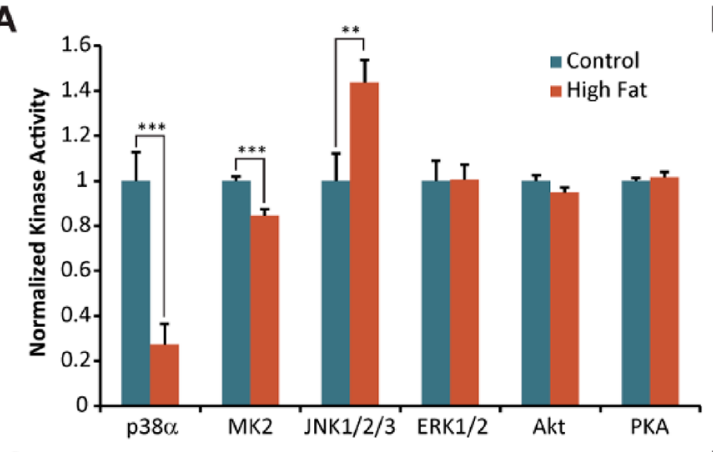

C

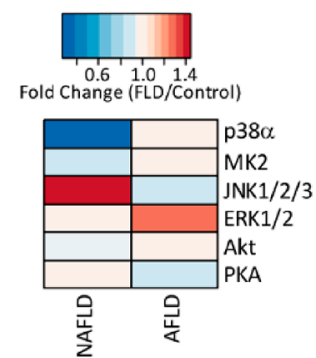

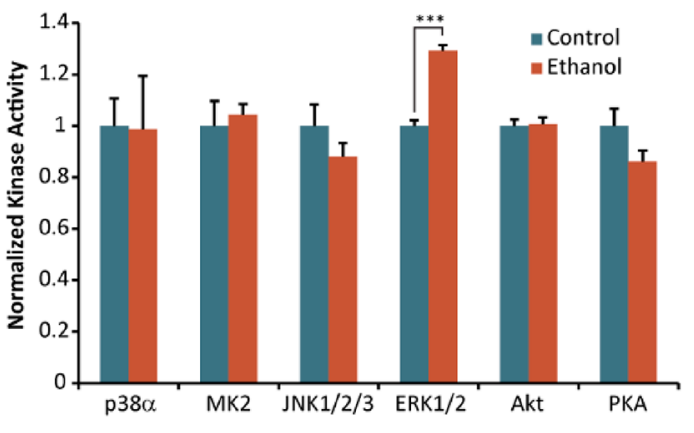

D

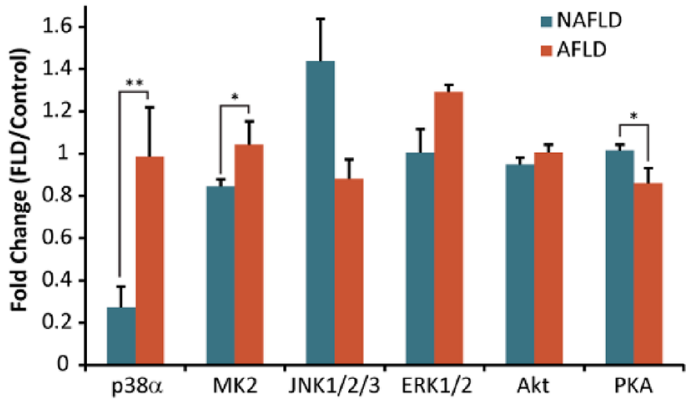

Figure 2. Kinase activity probes reveal distinct differences between diet-induced NAFLD and AFLD. Kinase activities in normal and diseased liver homogenates for (A) NAFLD $(n=12)$ and (B) AFLD $(n=6)$. Kinase activities are normalized to control samples. (C) Heat map showing clear differences in NAFLD vs AFLD kinase activities. Fold change is defined as the kinase activity in the fatty liver disease (FLD) samples relative to the corresponding control (normal) livers. (D) Statistically significant differences in p38 $\alpha$, MK2, and PKA activities distinguish NAFLD from AFLD. $p$ values from a two-tailed $t$ test are represented by one asterisk $(<0.05)$, two asterisks $(<0.01)$, or three asterisks $(<0.001)$. Error bars represent the standard error of the mean.

background subtraction of identical assays performed in the presence of the p38 $\alpha$ inhibitor SB203580 $(10 \mu \mathrm{M})$. Reaction slopes were determined from the initial linear portion of each assay. Heat maps of kinase activities were generated in $\mathrm{R}$.

Western Blotting. Lysates were separated on $12 \%$ SDSPAGE gels, and proteins were transferred to a nitrocellulose membrane. Blots were probed with primary antibodies for total p38 (Cell Signaling, 9212) or phospho-p38 (Cell Signaling, 9215). Primary antibody binding was detected using an HRPconjugated goat anti-rabbit (Pierce, 32460) secondary antibody. Blots were visualized by enhanced chemiluminescence (Pierce, 34075).

\section{RESULTS AND DISCUSSION}

NAFLD and AFLD Can Be Distinguished Using a Panel of Kinase Activity Probes. To investigate whether kinase activity probes could help facilitate parsing of NAFLD and AFLD, we chose to compare activity profiles of liver tissues obtained from animal models of NAFLD and AFLD. Although NAFLD and AFLD livers display indistinguishable histology (Figure S1), the etiology of each disease is different. Thus, we hypothesized that underlying signaling states may provide a basis for distinguishing NAFLD from AFLD. Accordingly, liver homogenates from rats fed a control diet or induced for NAFLD $(n=12)$ or AFLD $(n=6)$ development were obtained. These homogenates were assayed in 384-well plates using the panel of kinase activity probes (Figure 2A,B). Comparison of these profiles clearly indicated significant alterations in different MAPK signaling cascades in NAFLD versus AFLD. Specifically, we observed a $44 \pm 10 \%$ increase in JNK $1 / 2 / 3$ activity in NAFLD samples while ERK $1 / 2$ activity was increased by $29 \pm 2 \%$ in AFLD samples. These findings are consistent with previous reports of increased liver-associated JNK activity and phosphorylation in mouse models of NAFLD N1,32 $^{3}$ well as increased levels of phospho-ERK expression in human AFLD livers. ${ }^{33}$ In addition to these changes, we observed a $73 \pm 10 \%$ decrease in $\mathrm{p} 38 \alpha$ activity coupled with a $15 \pm 3 \%$ decrease in the activity of its downstream substrate, MK2, in NAFLD. To the best of our knowledge, this decrease in the activity of $\mathrm{p} 38 \alpha$ has not been previously reported. Western blotting indicated a decrease in the level of phosphorylation of total p38 $(\alpha, \beta, \gamma$, and $\delta)$, consistent with activity assays (Figure S2). These results imply the potential dysregulation of upstream protein kinase or phosphatase activity in NAFLD, which is currently under investigation in our laboratory. Previous work has identified p38 as a negative regulator of fat synthesis and storage in the liver. $^{34}$ Thus, deactivation of p38 signaling in NAFLD could allow for the accumulation of excess fat. Regardless of the consequences of these kinase activity perturbations, this data set confirms existing observations and comparison of NAFLD and AFLD kinase activities provides an activity signature that distinguishes between these disease states (Figure 2C). In particular, we observed statistically significant 3.6- and 1.2-fold increases in p38 $\alpha$ and MK2 activities, respectively, in AFLD and an $18 \pm 3 \%$ increase in PKA activity in NAFLD (Figure 2D). Thus, this panel of kinase chemosensors can be used to readily distinguish these histologically identical disease states.

Changes in Kinase Activity in Progressive NAFLD. Encouraged by the ability of kinase activity probes to distinguish histologically identical liver disease states, we next asked whether this probe panel could parse models of benign and progressive NAFLD. Accordingly, we set out to compare our diet-induced rat model of NAFLD, which does not progress to more serious disease phenotypes, to the wellestablished STAM mouse model of liver disease progression. ${ }^{30}$ 
A

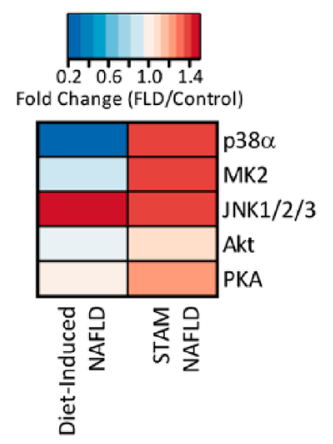

B

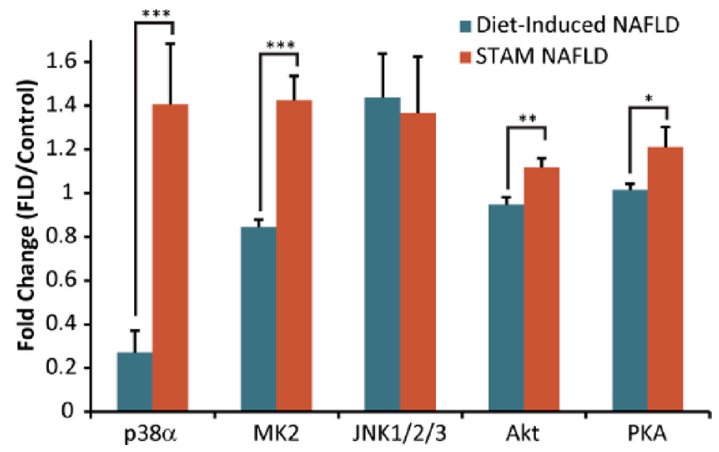

Figure 3. Changes in kinase activity differentiate diet-induced NAFLD and progressive STAM NAFLD. (A) Heat map showing differences in kinase enzymatic activity in diet-induced NAFLD vs STAM NAFLD (6 weeks). Fold change is defined as the kinase activity in the fatty liver disease (FLD) samples relative to the corresponding control (normal) livers. (B) Statistically significant differences in p38 $\alpha$, MK2, Akt, and PKA activities are observed in diet-induced NAFLD vs STAM NAFLD. $p$ values from a two-tailed $t$ test are represented by one asterisk $(<0.05)$, two asterisks $(<0.01)$, or three asterisks $(<0.001)$. Error bars represent the standard error of the mean.

The STAM model uses a two-hit protocol of initial treatment with streptozotocin (STZ) to induce the death of pancreatic $\beta$ cells, mimicking type I diabetes, followed by administration of a high-fat diet. The STAM model faithfully induces NAFLD that progresses to HCC with essentially 100\% fidelity (Figure S3). Liver homogenates were obtained from diet-induced NAFLD $(n=12)$ and STAM NAFLD $(n=5)$ models and interrogated using the panel of kinase activity probes (Figure S4A). These assays indicate clear differences in kinase signaling associated with the progressive, STAM NAFLD model [6 weeks (Figure 3A)]. Interestingly, STAM NAFLD displayed a 5.2-fold increase in $\mathrm{p} 38 \alpha$ activity compared to that of dietinduced NAFLD (Figure 3B). This observation indicates that the two-hit STAM model may lead to an increased level of inflammatory signaling through the p38 $\alpha$ axis, possibly potentiating a perturbed inflammatory state leading to disease progression. This hypothesis is currently under investigation in our laboratory. In addition, we observed statistically significant $69 \pm 11 \%, 18 \pm 4 \%$, and $19 \pm 9 \%$ increases in MK2, Akt, and PKA activities, respectively, in STAM NAFLD versus dietinduced NAFLD (Figure 3B). Together, these results indicate significant changes in inflammatory, survival, and metabolic signaling associated with the STAM model of NAFLD and provide a basis set for future experiments aimed at identifying factors that may potentiate NAFLD progression as well as approaches to block progression to NASH.

Longitudinal Kinase Activity during NAFLD Progression. To further investigate the utility of CSox-based activity probes for profiling changes associated with NAFLD progression, we obtained liver samples $(n=5)$ from the STAM mouse model corresponding to NAFLD (6 weeks), NASH ( 8 weeks), fibrosis (12 weeks), and HCC (20 weeks). Tissue homogenates for each of these samples as well as control animals were generated and normalized to the total protein content. Subsequent assays using CSox-based probes produced a temporal profile of kinase activity perturbations during disease progression (Figure 4 and Figure S4A-D). As expected, the activities of $\mathrm{p} 38 \alpha$ and its downstream effector, MK2, were coupled over time during disease progression (Figure 4 and Figure S4E). Activities of both enzymes peaked in NASH with $56 \pm 40 \%$ and $57 \pm 16 \%$ increases observed in p38 $\alpha$ and MK2 activities, respectively, relative to control samples. PKA and Akt activities began elevated by $21 \pm 9 \%$ and $12 \pm 4 \%$, respectively, in NAFLD and decreased in a linear

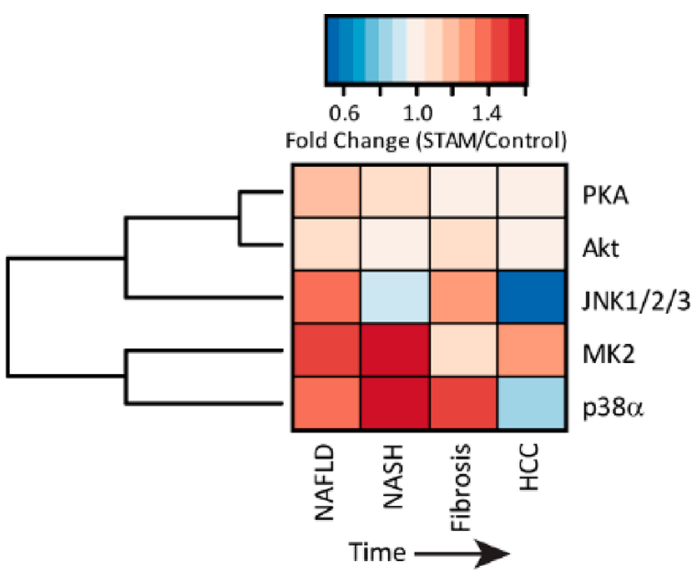

Figure 4. Heat map depicting temporal changes in kinase activity in the STAM model from NAFLD through HCC (treatment for 6-20 weeks). Fold change is defined as the kinase activity in the STAM livers relative to control livers.

fashion over time (Figure 4 and Figure S4F). Lastly, JNK1/2/3 activity displayed an oscillatory profile beginning with a $37 \pm$ $26 \%$ increase in NAFLD and ending with a $52 \pm 24 \%$ decrease in HCC (Figure 4 and Figure S4G). Taken together, this temporal profile provides a starting point for the analysis of kinase signaling dynamics during NAFLD progression. Current efforts are focused on utilizing additional CSox-based probes for kinases ${ }^{22,23,35,36}$ and phosphatases ${ }^{37-40}$ as well as small molecule probes for reactive oxygen species ${ }^{41,42}$ to further define potential changes associated with disease progression.

\section{CONCLUSION}

We have demonstrated the utility of panels of CSox-based kinase activity sensors for distinguishing histologically identical liver disease subtypes. These proof-of-principle experiments indicate the ability to parse NAFLD and AFLD as well as benign and progressive NAFLD. An initial temporal profile of kinase activity during NAFLD progression provides a starting point for further analysis of signaling changes associated with disease progression. The availability of a rapid, low-cost screening platform capable of distinguishing NAFLD from AFLD enables the potential development of clinical diagnostics 
and the identification of pathways for further investigation using small molecule inhibitors.

\section{ASSOCIATED CONTENT}

\section{S Supporting Information}

The Supporting Information is available free of charge on the ACS Publications website at DOI: 10.1021/acs.biochem.9b00547.

Histology images, Western blotting, and activity assays (PDF)

\section{AUTHOR INFORMATION}

\section{Corresponding Authors}

*E-mail: ccasey@unmc.edu.

*E-mail: eharris5@unl.edu.

*E-mail: cstains@virginia.edu.

\section{ORCID}

Edward N. Harris: 0000-0001-7045-1040

Cliff I. Stains: 0000-0002-3165-4539

\section{Funding}

This work was funded by National Institutes of Health (NIH) Grants R35GM119751 (C.I.S.) and R01HL130864 (E.N.H.), the Nebraska Research Initiative, the Proposed Center for Integrated Biomolecular Communication (CIBC), a seed grant from the Nebraska Center for the Prevention of Obesity Disease (P20GM104320), and the University of NebraskaLincoln. The content of this work is solely the responsibility of the authors and does not necessarily represent the official views of the NIH.

\section{Notes}

The authors declare no competing financial interest.

\section{ACKNOWLEDGMENTS}

The authors acknowledge the Research Instrumentation Facility and the Nebraska Center for Mass Spectrometry for assistance with characterization of probes.

\section{REFERENCES}

(1) Masarone, M., Federico, A., Abenavoli, L., Loguercio, C., and Persico, M. (2015) Non alcoholic fatty liver: epidemiology and natural history. Rev. Recent Clin. Trials 9, 126-133.

(2) Trauner, M., Arrese, M., and Wagner, M. (2010) Fatty liver and lipotoxicity. Biochim. Biophys. Acta, Mol. Cell Biol. Lipids 1801, 299310.

(3) Younossi, Z. M., Koenig, A. B., Abdelatif, D., Fazel, Y., Henry, L., and Wymer, M. (2016) Global epidemiology of nonalcoholic fatty liver disease-Meta-analytic assessment of prevalence, incidence, and outcomes. Hepatology 64, 73-84.

(4) Younossi, Z. M., Blissett, D., Blissett, R., Henry, L., Stepanova, M., Younossi, Y., Racila, A., Hunt, S., and Beckerman, R. (2016) The economic and clinical burden of nonalcoholic fatty liver disease in the United States and Europe. Hepatology 64, 1577-1586.

(5) Rasineni, K., Penrice, D. D., Natarajan, S. K., McNiven, M. A., McVicker, B. L., Kharbanda, K. K., Casey, C. A., and Harris, E. N. (2016) Alcoholic vs non-alcoholic fatty liver in rats: distinct differences in endocytosis and vesicle trafficking despite similar pathology. BMC Gastroenterol. 16, 27.

(6) Tannapfel, A., Denk, H., Dienes, H. P., Langner, C., Schirmacher, P., Trauner, M., and Flott-Rahmel, B. (2011) Histopathological diagnosis of non-alcoholic and alcoholic fatty liver disease. Virchows Arch. 458, 511-523.

(7) Kuhn, T., Nonnenmacher, T., Sookthai, D., Schubel, R., Quintana Pacheco, D. A., von Stackelberg, O., Graf, M. E., Johnson,
T., Schlett, C. L., Kirsten, R., Ulrich, C. M., Kaaks, R., Kauczor, H.-U. and Nattenmuller, J. (2018) Anthropometric and blood parameters for the prediction of NAFLD among overweight and obese adults. BMC Gastroenterol. 18, 113.

(8) Islami, F., Miller, K. D., Siegel, R. L., Fedewa, S. A., Ward, E. M., and Jemal, A. (2017) Disparities in liver cancer occurrence in the United States by race/ethnicity and state. Ca-Cancer J. Clin. 67, 273289.

(9) Xu, J. Q. (2018) Trends in liver cancer mortality among adults aged 25 and over in the United States, 2000-2016. Vol. NCHS Data Brief, no. 314, National Center for Health Statistics, Hyattsville, MD.

(10) Ajmera, V., Perito, E. R., Bass, N. M., Terrault, N. A., Yates, K. P., Gill, R., Loomba, R., Diehl, A. M., and Aouizerat, B. E. (2017) Novel Plasma Biomarkers Associated With Liver Disease Severity in Adults With Nonalcoholic Fatty Liver Disease. Hepatology 65, 65-77.

(11) Liu, K., Xu, W. Q., and Wong, V. W. S. (2017) Serum Biomarkers for Nonalcoholic Fatty Liver Disease: Are We There Yet? Hepatology 65, 8-11.

(12) Bishop, A. C., Ubersax, J. A., Petsch, D. T., Matheos, D. P., Gray, N. S., Blethrow, J., Shimizu, E., Tsien, J. Z., Schultz, P. G., Rose, M. D., Wood, J. L., Morgan, D. O., and Shokat, K. M. (2000) A chemical switch for inhibitor-sensitive alleles of any protein kinase. Nature 407, 395-401.

(13) Oldach, L., and Zhang, J. (2014) Genetically Encoded Fluorescent Biosensors for Live-Cell Visualization of Protein Phosphorylation. Chem. Biol. 21, 186-197.

(14) Ranjitkar, P., Brock, A. M., and Maly, D. J. (2010) Affinity Reagents that Target a Specific Inactive Form of Protein Kinases. Chem. Biol. 17, 195-206.

(15) Karginov, A. V., Zou, Y., Shirvanyants, D., Kota, P., Dokholyan, N. V., Young, D. D., Hahn, K. M., and Deiters, A. (2011) Light Regulation of Protein Dimerization and Kinase Activity in Living Cells Using Photocaged Rapamycin and Engineered FKBP. J. Am. Chem. Soc. 133, 420-423.

(16) Gonzalez-Vera, J. A. (2012) Probing the kinome in real time with fluorescent peptides. Chem. Soc. Rev. 41, 1652-1664.

(17) Zondlo, S. C., Gao, F., and Zondlo, N. J. (2010) Design of an Encodable Tyrosine Kinase-Inducible Domain: Detection of Tyrosine Kinase Activity by Terbium Luminescence. J. Am. Chem. Soc. 132, 5619-5621.

(18) Green, K. D., and Pflum, M. K. H. (2009) Exploring Kinase Cosubstrate Promiscuity: Monitoring Kinase Activity through Dansylation. ChemBioChem 10, 234-237.

(19) Wang, Q. Z., Cahill, S. M., Blumenstein, M., and Lawrence, D. S. (2006) Self-reporting fluorescent substrates of protein tyrosine kinases. J. Am. Chem. Soc. 128, 1808-1809.

(20) Shults, M. D., Janes, K. A., Lauffenburger, D. A., and Imperiali, B. (2005) A multiplexed homogeneous fluorescence-based assay for protein kinase activity in cell lysates. Nat. Methods 2, 277-283.

(21) Shults, M. D., and Imperiali, B. (2003) Versatile fluorescence probes of protein kinase activity. J. Am. Chem. Soc. 125, 1424814249.

(22) Beck, J. R., Harris, E. N., and Stains, C. I. (2017) Quantification of Cell Signaling Networks Using Kinase Activity Chemosensors. Methods Mol. Biol. 1636, 61-70.

(23) Beck, J. R., Peterson, L. B., Imperiali, B., and Stains, C. I. (2014) Quantification of protein kinase enzymatic activity in unfractionated cell lysates using CSox-based sensors. Curr. Protoc. Chem. Biol. 6, 135-156.

(24) Lukovic, E., Gonzalez-Vera, J. A., and Imperiali, B. (2008) Recognition-domain focused chemosensors: Versatile and efficient reporters of protein kinase activity. J. Am. Chem. Soc. 130, 1282112827.

(25) Zamora-Olivares, D., Kaoud, T. S., Jose, J., Ellington, A., Dalby, K. N., and Anslyn, E. V. (2014) Differential Sensing of MAP Kinases Using SOX-Peptides. Angew. Chem., Int. Ed. 53, 14064-14068.

(26) Lukovic, E., Vogel Taylor, E., and Imperiali, B. (2009) Monitoring protein kinases in cellular media with highly selective chimeric reporters. Angew. Chem., Int. Ed. 48, 6828-6831. 
(27) Peterson, L. B., Yaffe, M. B., and Imperiali, B. (2014) Selective Mitogen Activated Protein Kinase Activity Sensors through the Application of Directionally Programmable D Domain Motifs. Biochemistry 53, 5771-5778.

(28) Stains, C. I., Tedford, N. C., Walkup, T. C., Lukovic, E., Goguen, B. N., Griffith, L. G., Lauffenburger, D. A., and Imperiali, B. (2012) Interrogating Signaling Nodes Involved in Cellular Transformations Using Kinase Activity Probes. Chem. Biol. 19, 210-217.

(29) Zamora-Olivares, D., Kaoud, T. S., Dalby, K. N., and Anslyn, E. V. (2013) In-Situ Generation of Differential Sensors that Fingerprint Kinases and the Cellular Response to Their Expression. J. Am. Chem. Soc. 135, 14814-14820.

(30) Fujii, M., Shibazaki, Y., Wakamatsu, K., Honda, Y., Kawauchi, Y., Suzuki, K., Arumugam, S., Watanabe, K., Ichida, T., Asakura, H., and Yoneyama, H. (2013) A murine model for non-alcoholic steatohepatitis showing evidence of association between diabetes and hepatocellular carcinoma. Med. Mol. Morphol. 46, 141-152.

(31) Hirosumi, J., Tuncman, G., Chang, L. F., Gorgun, C. Z., Uysal, K. T., Maeda, K., Karin, M., and Hotamisligil, G. S. (2002) A central role for JNK in obesity and insulin resistance. Nature 420, 333-336.

(32) Schattenberg, J. M., Singh, R., Wang, Y., Lefkowitch, J. H., Rigoli, R. M., Scherer, P. E., and Czaja, M. J. (2006) Jnk1 but not jnk2 promotes the development of steatohepatitis in mice. Hepatology 43, $163-172$.

(33) Choi, E., Kim, W., Joo, S. K., Park, S., Park, J. H., Kang, Y. K., Jin, S.-Y., and Chang, M. S. (2018) Expression patterns of STAT3, ERK and estrogen-receptor $\alpha$ are associated with development and histologic severity of hepatic steatosis: a retrospective study. Diagn. Pathol. 13, 23.

(34) Xiong, Y., Collins, Q. F., An, J., Lupo, E., Jr., Liu, H. Y., Liu, D., Robidoux, J., Liu, Z., and Cao, W. (2007) p38 mitogen-activated protein kinase plays an inhibitory role in hepatic lipogenesis. J. Biol. Chem. 282, 4975-4982.

(35) Beck, J. R., Zhou, X., Casey, G. R., and Stains, C. I. (2015) Design and evaluation of a real-time activity probe for focal adhesion kinase. Anal. Chim. Acta 897, 62-68.

(36) Kelly, M. I., Bechtel, T. J., Reddy, D. R., Hankore, E. D., Beck, J. R., and Stains, C. I. (2015) A real-time, fluorescence-based assay for Rho-associated protein kinase activity. Anal. Chim. Acta 891, 284290.

(37) Beck, J. R., Lawrence, A., Tung, A. S., Harris, E. N., and Stains, C. I. (2016) Interrogating Endogenous Protein Phosphatase Activity with Rationally Designed Chemosensors. ACS Chem. Biol. 11, 284290.

(38) Beck, J. R., Truong, T., and Stains, C. I. (2016) Temporal Analysis of PP2A Phosphatase Activity During Insulin Stimulation Using a Direct Activity Probe. ACS Chem. Biol. 11, 3284-3288.

(39) Casey, G. R., and Stains, C. I. (2018) Interrogating Protein Phosphatases with Chemical Activity Probes. Chem. - Eur. J. 24, $7810-7824$.

(40) Casey, G. R., Beck, J. R., and Stains, C. I. (2019) Design and synthesis of fluorescent activity probes for protein phosphatases. Methods Enzymol. 622, 29-53.

(41) Zhou, X., Lesiak, L., Lai, R., Beck, J. R., Zhao, J., Elowsky, C. G., Li, H., and Stains, C. I. (2017) Chemoselective Alteration of Fluorophore Scaffolds as a Strategy for the Development of Ratiometric Chemodosimeters. Angew. Chem., Int. Ed. 56, 4197-4200.

(42) Zhou, X., Lai, R., Beck, J. R., Li, H., and Stains, C. I. (2016) Nebraska Red: a phosphinate-based near-infrared fluorophore scaffold for chemical biology applications. Chem. Commun. 52, 12290-12293. 


\section{Supporting Information}

\section{A Panel of Protein Kinase Chemosensors Distinguishes Different Types of Fatty Liver Disease}

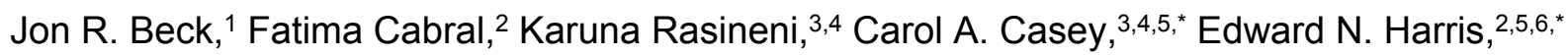
and Cliff I. Stains $\mathbf{s}^{1,5,6,7, *}$

${ }^{1}$ Department of Chemistry, University of Nebraska - Lincoln, Lincoln, NE 68588, USA ${ }^{2}$ Department of Biochemistry, University of Nebraska - Lincoln, Lincoln, NE 68588, USA ${ }^{3}$ Division of Gastroenterology-Hepatology, University of Nebraska Medical Center, Omaha, NE 68198, USA

${ }^{4}$ Research Service, Veterans' Affairs, Nebraska-Western lowa Health Care System, Omaha, NE 68105, USA

${ }^{5}$ Nebraska Center for Integrated Biomolecular Communication, University of Nebraska Lincoln, Lincoln, NE 68588, USA

${ }^{6}$ Cancer Genes and Molecular Regulation Program, Fred \& Pamela Buffet Cancer Center, University of Nebraska Medical Center, Omaha NE 68198, USA

${ }^{7}$ Department of Chemistry, University of Virginia, Charlottesville, VA, 22904

`E-mail: ccasey@unmc.edu (C.A.C.), eharris5@unl.edu (E.N.H.), cstains@virginia.edu (C.I.S.)

\section{Table of Contents}

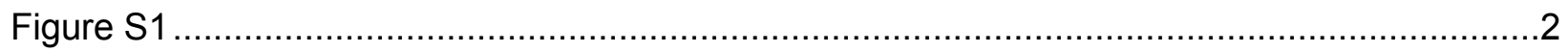

Figure S2

Figure S3

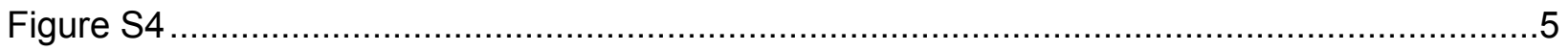


Figure S1
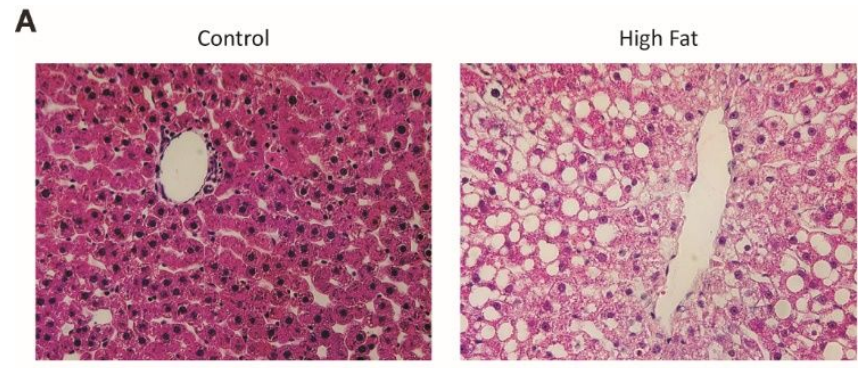

B
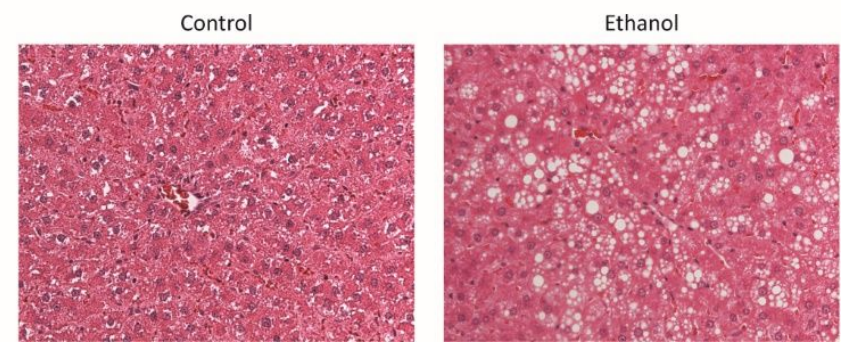

Indistinguishable histology of NAFLD and AFLD. H\&E staining for NFALD (A) and AFLD (B) livers. 


\section{Figure $\mathbf{S 2}$}

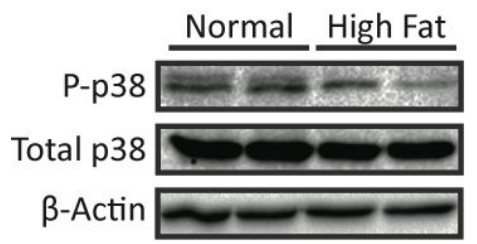

NAFLD livers display decreased phosphorylation of p38. Reduced phosphorylation of the p38 (P-p38) activation loop is observed in NAFLD (High Fat) versus control (Normal) livers. Total p38 staining indicates that levels of p38 do not change appreciably in each sample. Total p38 and P-p38 antibodies recognize $\alpha, \beta, \gamma$, and $\delta$ isoforms of p38. $\beta$-actin staining demonstrates equivalent protein loading in each lane. 
Figure \$3

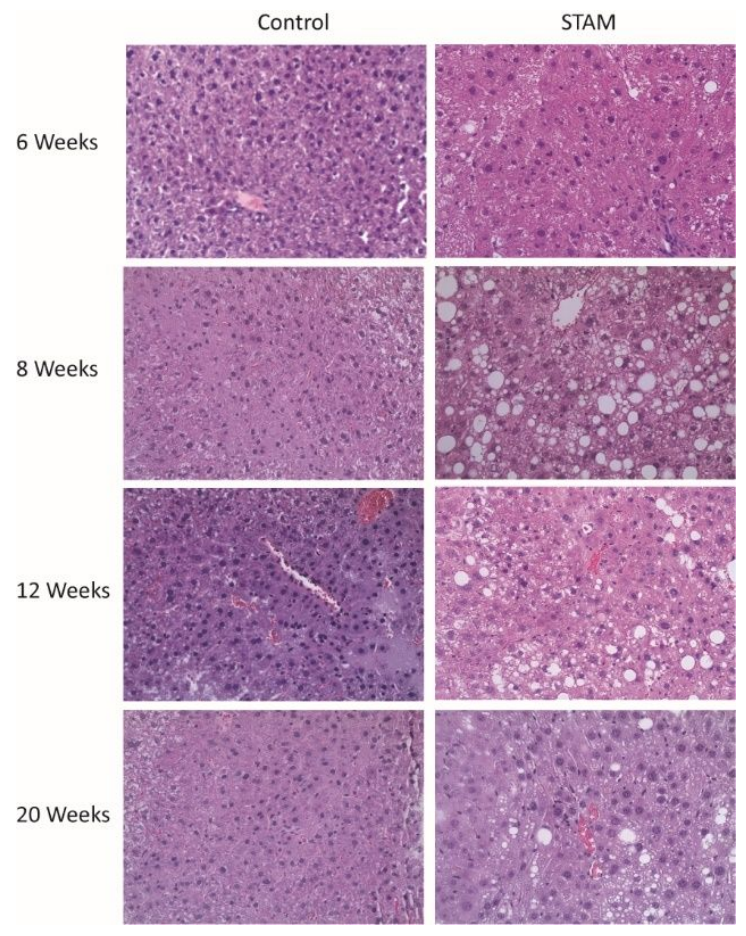

Progression of the STAM model through increasingly severe stages of liver disease. H\&E staining of livers from animals fed a control diet as well as STAM animals at varying time points corresponding to NAFLD (6 weeks), NASH (8 weeks), fibrosis (12 weeks), and HCC (20 weeks). 
Figure $\$ 4$

A

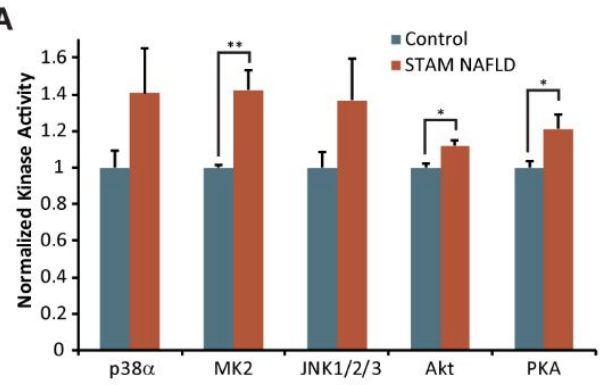

C

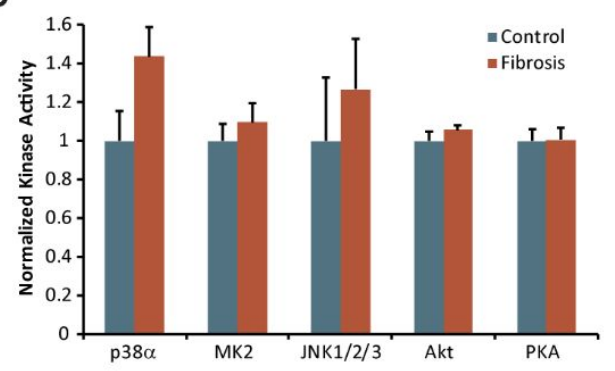

B

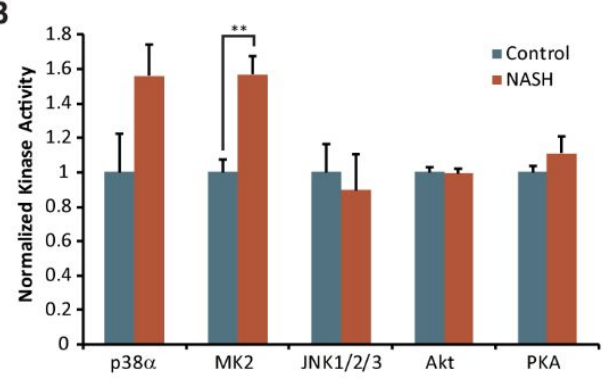

D

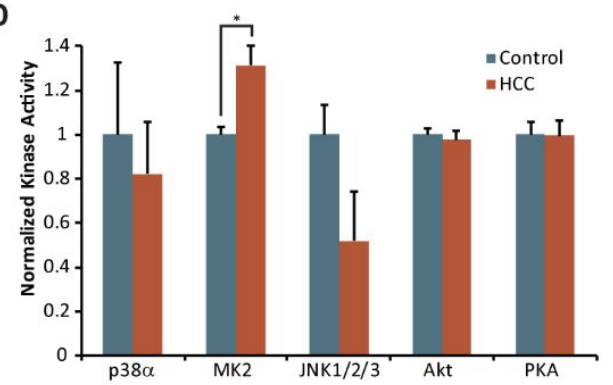

$\mathrm{E}$

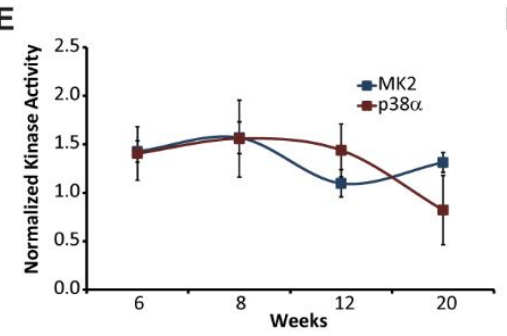

$\mathrm{F}$

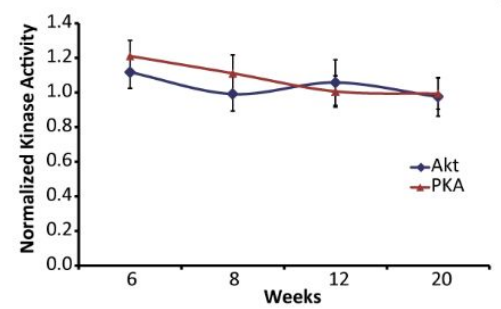

G

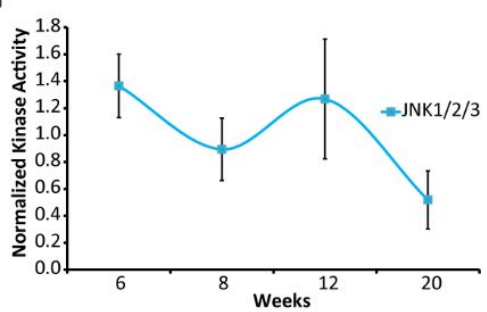

Changes in kinase activity in the STAM model. Kinase activities for STAM NAFLD $(A, n=5)$, $\mathrm{NASH}(B, n=5)$, fibrosis $(C, n=5)$, and $\operatorname{HCC}(D, n=5)$ relative to control animals $(n=5)$ are shown. Significant increases in MK2 (43\%), Akt (12\%), and PKA (21\%) activities are observed in STAM NAFLD. A significant increase in MK2 activity in NASH (57\%) and HCC (31\%) was observed. Kinase activities for MK2 and p38a (E), Akt and PKA (F), and JNK (G) are clustered according to similar trends in activity during disease progression. STAM $(n=5)$ samples are normalized to control animals $(n=5)$. Activities are shown versus time for STAM NAFLD (6 week), NASH (8 week), fibrosis (12 week), and HCC (20 week) samples. P-values from a twotailed t-test are represented by ${ }^{*}(<0.05)$ or ${ }^{* *}(<0.01)$. Error bars represent sem. 